\title{
Dynamic Parameter Identification of Hydrodynamic Bearing-Rotor System
}

\author{
Zhiqiang Song and Yunhe Liu \\ State Key Laboratory Base of Eco-Hydraulic Engineering in Arid Area, Xian University of Technology, Xian 710048, China \\ Correspondence should be addressed to Zhiqiang Song; szhiq2004@126.com
}

Received 27 March 2015; Accepted 4 August 2015

Academic Editor: Mickaël Lallart

Copyright (C) 2015 Z. Song and Y. Liu. This is an open access article distributed under the Creative Commons Attribution License, which permits unrestricted use, distribution, and reproduction in any medium, provided the original work is properly cited.

\begin{abstract}
A new method called modal parameter genetic time domain identification was employed to study the characteristics of the bearingrotor system. A multifrequency signal decomposition technology to identify the main components of the measured signal and reject the image mode produced by noise has been used. The first- and second-order natural frequency and damping ratios of the shaft system are identified. Furthermore, because of the deficiency of the traditional least square method, a new genetic identification method to identify the bearing dynamic characteristic parameters has been proposed. The method has been effective albeit with few testing points and operation cases. The derivation of oil-film dynamic coefficients could also provide a basis for shaft system natural vibration characteristic and vibration response analysis. Using the identified dynamic coefficients as the supporting condition, the shaft system modal characteristics were studied. The calculated first- and second-order natural frequencies match quite well those obtained from the modal parameter identification. It was proved that the modal parameter and physical parameter identification methods utilized in this paper are reasonable.
\end{abstract}

\section{Introduction}

Recently, the finite element methods have been widely used in the study on vibration characteristics of rotating machines such as the water turbine generator set shaft system $[1,2]$. However, due to the uncertainty of the boundary condition or dynamic parameters, the simulation results were sometimes far from practical. The system identification theory deals with the vibration problem depending on the theory analysis and prototype testing. It could obtain the boundary condition or dynamic physical parameters according to the measured structure response. The identification could provide the rationality of boundary condition or dynamic parameters for further response characteristic analysis of the rotating machine.

To date, system identification can generally be classified into two categories: (a) identification of modal parameters of the system and (b) identification of physical parameters of the system. Modal parameters reflect the natural vibration characteristics of the system better than physical parameters. The number of modal parameters is also fewer than that of physical parameters. Hence, the modal parameter identification is the basis of system identification. Over the decades, modal parameter identification has become well accepted and widely used in the study of vibration stability of rotating machines. However, the traditional method of frequency domain identification must require a significant number of tests to obtain the frequency response functions. Obtaining the frequency response function may be timeconsuming and costly; the identification would not be carried out without the function. In the recent past, it has been observed that time domain identification has played a significant role in the rapid resolution for modal parameters [3-5].

Based on the linear structure vibration mode superposition theory, the time domain identification method was proposed to obtain the modal parameters from the measured structure response signals. This method could identify the modal parameters of large structures that work only under environment excitation. It did not need system input information. It had no influence on the normal operation of the structure or equipment. Hence, this method is very suitable for modal identification of continuously operating equipment such as the water turbine generator set shaft system. 
Compared with modal parameter identification, the physical parameters were much more direct and visual. The physical parameters obtained by identification are the basis of structural characteristic analysis and vibration response resolutions. The physical parameters that need to be identified for the generator set shaft system are just the oil-film dynamic characteristic coefficients of the journal bearings. Rotating machines are supported by bearings, which play a vital role in determining the behavior of rotating systems under the action of dynamic loads. One of the most important factors governing the vibration characteristics of rotating machinery is the parameters of bearing dynamics. Although the importance of rotor support dynamic stiffness is generally well recognized by the design engineer, it is often the case that theoretical models available for predicting it are insufficiently accurate or are accurate only in very specific cases. Moreover, the stiffness and damping characteristic coefficients are greatly dependent on many physical and mechanical parameters such as lubricant temperature, bearing clearance and load, journal speed, and machine misalignment in the system, and these are difficult to obtain accurately under test conditions. Uncertainties about machine parameters can render the results obtained with the best theoretical methods inaccurate in the study of the behavior of fluidfilm journal bearings. Owing to this, it may be very useful to determine the unbalance and bearing dynamic stiffness by means of identification methods based on experimental data and machine models.

Many researchers have worked on the identification of oil-film coefficients [6-8]. Yao et al. [9] have utilized the rotating machinery analysis to obtain the critical speed and oilfilm stiffness. Because the critical speed of water electric units is higher than the rated speed or working speed, this method is not suitable for water electric units. Lees and Friswell [10] have presented a method to evaluate the state of unbalance and bearing coefficients of a rotating machine using the measured pedestal vibration. Subsequently, Edwards et al. [11] have presented experimental verification of the method to evaluate the unbalance and bearing coefficients of a rotating machine. Tiwari and Chakravarthy [12] have proposed an identification algorithm for simultaneous estimation of residual unbalances and bearing dynamic parameters. The algorithm uses standard condensation to overcome the difficulty with too much response freedom that needs to be measured. The identification parameters matched quite well the parameters assumed for the simulation of responses. Zhao et al. [13] have proposed an experimental method to identify linear oil-film coefficients and presented the characteristics under various operational conditions. They had pointed out that linear oil-film dynamic coefficients are sensitive to excitation amplitude. Of course, their linear oil-film model would be invalid if the condition of small perturbation is not satisfied. Meruane and Pascual [14] have proposed a framework for numerical identification of nonlinear fluid-film-bearing parameters from larger journal orbital motion. Nonlinear coefficients are defined by third-order Taylor expansion of bearing reaction forces and are evaluated through least mean square in time domain technique. Lou and $\mathrm{Bi}[15,16]$ have also utilized the least square method to obtain oil-film coefficients. This method needs data from different running cases to satisfy the request about the equation number. However, if the operation cases change greatly, the oil-film coefficients will vary greatly, which may lead to the premise that the identification formula is not satisfied any more. Recently, Tiwari et al. $[17,18]$ have provided a review of the identification procedures applied to bearing and seal models, the existing experimental techniques for acquiring the measurement data from the rotor-bearing-seal test rigs, and theoretical procedures to extract the relevant bearing and seal dynamics parameters and estimate-associated parameter uncertainties. They had concluded that the synchronous unbalance response, which can easily be obtained from the run-down/up of large turbo machines, should be exploited more for the identification of bearing dynamics parameters along with the estimation of residual unbalance.

The organization of the rest of the paper is as follows: theory of the modal parameter identification method is described in Section 2; a newly developed multifrequency signal decomposition technology has been used to identify the main components of measured signal and reject the image mode produced by noise. Section 3 presents a new genetic identification method for the journal bearing dynamic characteristic parameters; using the dynamic coefficients obtained, the shaft system modal characteristics can be then studied using finite element method; the calculated first- and second-order natural frequencies matched quite well those obtained from modal parameter identification. The conclusion is presented in Section 4.

\section{Modal Parameter Genetic Time Domain Identification}

2.1. Algorithm for Genetic Time Domain Identification. For a system with $n$ freedoms, the equation of free motion is given as

$$
[M]\{\ddot{x}\}+[C]\{\dot{x}\}+[K]\{x\}=0 .
$$

Let $\{x\}=[\varphi]\left\{e^{\lambda t}\right\}$, where $[\varphi]$ is called the free response amplitude matrix. Hence, the Eigenvalue $\lambda$ could be expressed as

$$
\lambda_{i}=-\xi_{i} \omega_{i} \pm j \omega_{d i} \quad(i=1,2, \ldots, n),
$$

where $\xi_{i}$ is damping, $\omega_{i}$ is vibration frequency without damping, while $\omega_{d i}$ is vibration frequency with damping, and $\omega_{d i}=\omega_{i} \sqrt{1-\xi_{i}^{2}}$.

The coordinates of transformation of displacement vector $\{x\}$ are given by

$$
\{x\}=\sum_{i=1}^{n} \varphi_{i} y_{i}=[\Phi]\{y\} .
$$

Substituting (3) into (1),

$$
\{\ddot{y}\}+\operatorname{diag}\left[\sigma_{i}\right]\{\dot{y}\}+\operatorname{diag}\left[\omega_{0 i}\right]\{y\}=0 .
$$

Equation (4) could be solved taking the initial conditions into account:

$$
y_{i}=Y_{i} e^{-\xi_{i} \omega_{i} t} \sin \left(\omega_{d i} t+\theta_{i}\right)
$$


where $Y_{i}$ and $\theta_{i}$ are constants depending on the initial conditions:

$$
\begin{aligned}
Y_{i} & =\sqrt{y_{0 i}^{2}+\left(\frac{\dot{y}_{0 i}+\xi_{i} \omega_{i} y_{0 i}}{\omega_{d i}}\right)^{2}} \\
\theta_{i} & =\arctan \frac{\omega_{d i} y_{0 i}}{\dot{y}_{0 i}+\xi_{i} \omega_{i} y_{0 i}} .
\end{aligned}
$$

Substituting (5) into (3),

$$
\begin{aligned}
x & =\sum_{i=1}^{n} \varphi_{i} Y_{i} e^{-\sigma_{i} t} \sin \left(\omega_{d i} t+\theta_{i}\right) \\
& =\sum_{i=1}^{n} D_{i} e^{-\sigma_{i} t} \sin \left(\omega_{d i} t+\theta_{i}\right),
\end{aligned}
$$

where $\sigma_{i}=\xi_{i} \omega_{i}$ and $D_{i}=\varphi_{i} Y_{i}$.

The precision request could be satisfied well as long as the modal displacement responses of first few orders are taken.

The modal parameter genetic identification method is based on the system identification and genetic optimization theory [19]. This method utilizes the free vibration response measurement signals of the shaft system. The chromosomes are used to express the modal parameters such as natural frequencies and damping ratios. The parameters that made the objective function optimal are just the structural modal parameters.

The objective function could be expressed by an equation that includes the measured response and calculated response:

$$
\min L\left(\xi_{i}, \omega_{i}\right)=\left[x(t)-\sum_{i=1}^{M} D_{i} e^{-\sigma_{i} t} \sin \left(\omega_{d i} t+\theta_{i}\right)\right]^{2},
$$

where $x(t)$ is the measured response.

$\sum_{i=1}^{M} D_{i} e^{-\sigma_{i} t} \sin \left(\omega_{d i} t+\theta_{i}\right)$ is the calculated response ( $M$ is the modal order number).

2.2. Multifrequency Signal Process. Vibration signals are always composed of many frequency components. The noise would produce the image mode with poor identification accuracy. Using the signal decomposition technology, a new multifrequency identification algorithm to identify the main components of a multifrequency signal and reject the image mode produced by noise has been proposed.

A random process could be expressed as follows:

$$
x(n)=\sum_{i=1}^{M} A_{i} e^{j\left(\omega_{i} n+\varphi_{i}\right)}+w(n),
$$

where $\sum_{i=1}^{M} A_{i} e^{j\left(\omega_{i} n+\varphi_{i}\right)}$ is the signal composed by $M$ sine waves, the signal determined order is to make sure of the number of sine waves $M$, and $w(n)$ is additive white noise.

The autocorrelation function of signal $x(n)$ could be calculated from

$$
R_{x}(m)=\sum_{i=1}^{M} A_{i}^{2} e^{j \omega_{i} m}+\sigma_{w}^{2} \delta(m),
$$

where $m=0,1, \ldots, N-1 ; \sigma_{w}$ is the square variance of the random signal. Now, define the signal vectors

$$
e_{i}=\left[\begin{array}{llll}
1 & e^{j \omega_{i}} & \cdots & e^{j(N-1) \omega_{i}}
\end{array}\right]^{T} \quad(i=1,2, \ldots, M),
$$

where $\omega_{i}$ is the frequency of $M$ sine signals.

The autocorrelation matrix of signal $x(n)$ is expressed by the summation of signal autocorrelation matrix and noise autocorrelation matrix:

$$
R_{x}=R_{s}+R_{w}=\sum_{i=1}^{M} A_{i}^{2} e_{i} e_{i}^{H}+\sigma_{w}^{2} I_{N N},
$$

where $\sigma_{w}^{2} I_{N N}$ is the noise autocorrelation matrix and $I_{N N}$ is an $N \times N$-dimensional unit matrix.

$R_{s}$ could be expressed by its Eigenvalues and Eigenvectors:

$$
R_{s} v_{i}=\lambda_{i} v_{i} \quad(i=1,2, \ldots, N) .
$$

Generally, the rank of signal autocorrelation matrix $R_{s}$ (i.e., $M$ ) is less than that of its dimension number $N$. Hence, matrix $R_{s}$ has $M$ Eigenvalues that are nonzero:

$$
R_{s}=\sum_{i=1}^{M} \lambda_{i} v_{i} v_{i}^{H} .
$$

$R_{w}$ also could be expressed by its Eigenvalues and Eigenvectors. Because all its Eigenvalues are 1, any vectors could be regarded as its Eigenvectors $R_{w}$ :

$$
R_{w}=\sigma_{w}^{2} I_{N N}=\sigma_{w}^{2} \sum_{i=1}^{N} v_{i} v_{i}^{H} .
$$

Substituting (14) and (15) into (12),

$$
\begin{aligned}
R_{x} & =R_{s}+R_{w}=\sum_{i=1}^{M} \lambda_{i} v_{i} v_{i}^{H}+\sigma_{w}^{2} \sum_{i=1}^{N} v_{i} v_{i}^{H} \\
& =\sum_{i=1}^{M}\left(\lambda_{i}+\sigma_{w}^{2}\right) v_{i} v_{i}^{H}+\sum_{i=M+1}^{N} \sigma_{w}^{2} v_{i} v_{i}^{H},
\end{aligned}
$$

where $v_{M+1}, v_{M+2}, \ldots, v_{N}$ are noise Eigenvectors, which have the same Eigenvalues $\sigma_{w}^{2} ; v_{1}, v_{2}, \ldots, v_{M}$ are signal Eigenvector whose Eigenvalues are $\lambda_{1}+\sigma_{w}^{2}, \lambda_{2}+\sigma_{w}^{2}, \ldots, \lambda_{M}+\sigma_{w}^{2}$. It could be seen that white noise has an influence on sine signal Eigenvalues. Because the signal vectors $e_{i}(i=1,2, \ldots, M)$ and noise Eigenvectors $v_{M+1}, v_{M+2}, \ldots, v_{N}$ are orthogonal [20], their linear combination is also orthogonal:

$$
e_{i}^{H}\left(\sum_{j=M+1}^{N} a_{j} v_{j}\right)=0, \quad(i=1,2, \ldots, M) .
$$

Let $e(\omega)=e\left(\omega_{i}\right)=\left[\begin{array}{llll}1 & e^{j \omega_{i}} & \cdots & e^{j(N-1) \omega_{i}}\end{array}\right]^{T}=e_{i}$. So

$$
e^{H}(\omega)\left[\sum_{j=M+1}^{N} a_{j} v_{j} v_{j}^{H}\right] e(\omega)=0 .
$$




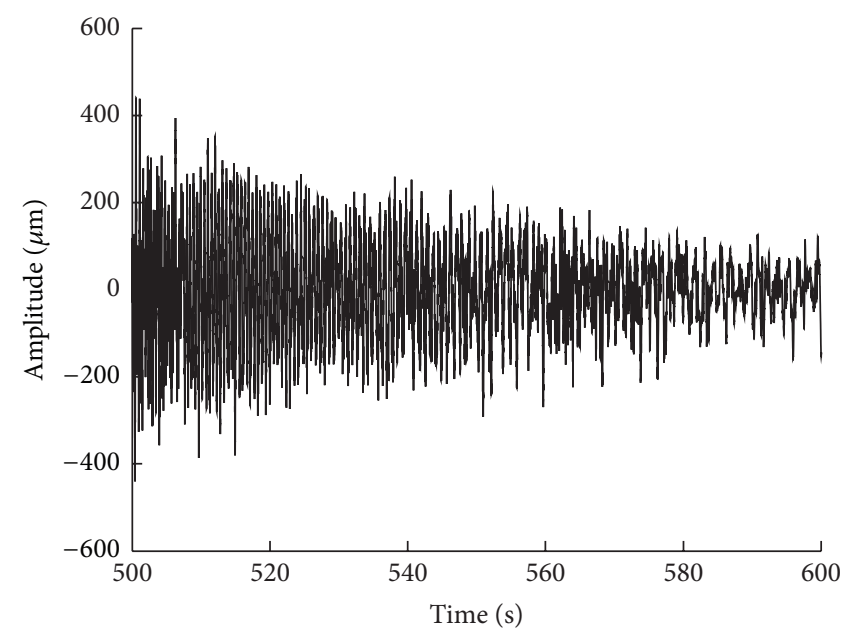

FIGURE 1: Vibration response of the shaft system.

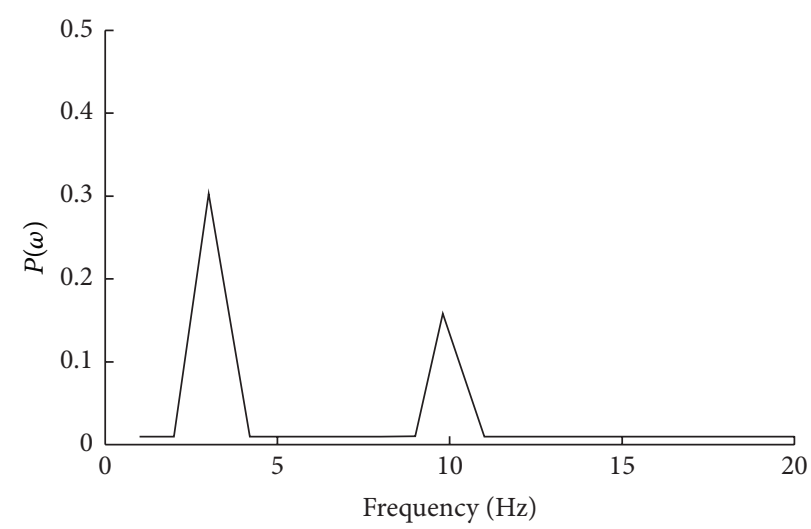

FIGURE 2: Vibration order of the shaft system.

Let $a_{j}=1(j=M+1, M+2, \ldots, N)$. Now the function $P_{x}(\omega)$ could be defined similarly to the power spectrum function:

$$
P_{x}(\omega)=\frac{1}{e^{H}(\omega)\left(\sum_{j=M+1}^{N} v_{j} v_{j}^{H}\right) e(\omega)}
$$

The function-independent variable $\omega$ is just a sine frequency of the signal when the function has a wave peak. It seems that the identification frequency $\omega$ could make the function $P_{x}(\omega)$ tend to infinity. In fact, the Eigenvectors $v_{i}$ would be obtained from the approximate autocorrelation matrix. Because of the estimate error, the function $P_{x}(\omega)$ only has a finite peak.

Now $M$ is the order number of the signal that needs to be determined. Assuming $M=1,2,3, \ldots$, find out whether the function $P_{x}(\omega)$ has a peak in the possible frequency domain. If the function $P_{x}(\omega)$ has a peak, the corresponding $M$ is just the sine wave number in the signal. Thus, the vibration modal order number of the structure could be obtained. For the hydroelectric unit shaft system, the natural frequency region is about $0 \sim 150 \mathrm{~Hz}$ and $M$ may be $1 \sim 5$. Hence, the computation cost is not very high.
2.3. Modal Parameter Identification of the Shaft System. Figure 1 shows the free vibration response signal of a factual unit shaft at the turbine guide bearing location at run-down operating condition. The new multifrequency identification algorithm was used to identify the main components of a multifrequency signal and reject the image mode produced by noise. The result is shown in Figure 2. It can be seen that the signal includes two orders of the vibration modals. The modal parameters were identified using the genetic time domain identification method. The identification results show that the first two-order natural frequencies are $3.9 \mathrm{~Hz}$ and $9.7 \mathrm{~Hz}$, and the first two-order damping ratios are $2.23 \%$ and $1.56 \%$.

\section{Bearing Dynamics Parameter Identification}

The identification of bearing dynamics parameters is helpful to understand the shaft system support conditions at normal operation cases. This may provide the basis for the study of shaft system modal characteristics and vibration responses.

The dynamic differential equation could be expressed as follows considering the mass unbalanced force and bearing oil-film force:

$$
[M]\{\ddot{Q}\}+[D]\{\dot{Q}\}+[K]\{Q\}=\{h\}-\{F\},
$$


where $\{Q\}$ is the node displacement vector; $\{h\}$ is the mass unbalanced force vector; $\{F\}$ is the bearing oil-film force matrix.

The response of the rotor system could be expressed by the superposition of multiple sine and cosine components:

$$
\{Q(t)\}=\sum_{i=1}^{n}\left[U^{i} \cos \left(J_{i} \omega t\right)+V^{i} \sin \left(J_{i} \omega t\right)\right],
$$

where $J_{i}(i=1,2, \ldots, n)$ is the ratio of a sine or cosine component to the rated rotating speed, which could be only a plus integer or rational fraction; $n$ is the total number of humorous wave components. Now, the mass unbalanced force could be written as

$$
\{h(t)\}=\sum_{i=1}^{n}\left[h_{c}^{i} \cos \left(J_{i} \omega t\right)+h_{s}^{i} \sin \left(J_{i} \omega t\right)\right] .
$$

Arbitrary journal bearing node displacement is given by

$$
\begin{aligned}
\left\{Q_{j}(t)\right\} & =\left\{\begin{array}{l}
x_{j} \\
y_{j}
\end{array}\right\} \\
& =\left\{\begin{array}{l}
\sum_{i=1}^{n}\left[x_{c j}^{i} \cos \left(J_{i} \omega t\right)+x_{s j}^{i} \sin \left(J_{i} \omega t\right)\right] \\
\sum_{i=1}^{n}\left[y_{c j}^{i} \cos \left(J_{i} \omega t\right)+x_{s j}^{i} \sin \left(J_{i} \omega t\right)\right]
\end{array}\right\} \\
& =\sum_{i=1}^{n}\left[U_{j}^{i} \cos \left(J_{i} \omega t\right)+V_{j}^{i} \sin \left(J_{i} \omega t\right)\right],
\end{aligned}
$$

where $j=1,2, \ldots, l$ denotes the journal bearing number, the total number of journal bearings being $l$.

The derivate $\left\{\dot{Q}_{j}(t)\right\}$ could be expressed as

$$
\begin{aligned}
& \left\{\dot{Q}_{j}(t)\right\} \\
& =\sum_{i=1}^{n}\left[U_{j}^{i} \sin \left(J_{i} \omega t\right) \cdot\left(-J_{i} \omega\right)+V_{j}^{i} \cos \left(J_{i} \omega t\right) \cdot\left(J_{i} \omega\right)\right] .
\end{aligned}
$$

The corresponding bearing oil-film force is given by

$$
\begin{aligned}
\left\{F_{j}\right\} & =\left[K_{j}\right]\left\{Q_{j}\right\}+\left[D_{j}\right]\left\{\dot{Q}_{j}\right\} \\
& =\left[\begin{array}{ll}
k_{x x j} & k_{x y j} \\
k_{y x j} & k_{y y j}
\end{array}\right]\left\{\begin{array}{l}
x_{j} \\
y_{j}
\end{array}\right\}+\left[\begin{array}{ll}
d_{x x j} & d_{x y j} \\
d_{y x j} & d_{y y j}
\end{array}\right]\left\{\begin{array}{c}
\dot{x}_{j} \\
\dot{y}_{j}
\end{array}\right\} .
\end{aligned}
$$

Let

$$
\begin{aligned}
& \left\{k_{j}\right\}=\left\{\begin{array}{llll}
k_{x x j} & k_{x y j} & k_{y x j} & k_{y y j}
\end{array}\right\}^{T}, \\
& \left\{d_{j}\right\}=\left\{\begin{array}{llll}
d_{x x j} & d_{x y j} & d_{y x j} & d_{y y j}
\end{array}\right\}^{T} .
\end{aligned}
$$

The oil-film force could be expressed as

$$
\begin{aligned}
& \left\{F_{j}\right\}=\sum_{i=1}^{n}\left[\left(\left[Y_{c j}^{i}\right]\left\{k_{j}\right\}+\left[Y_{s j}^{i}\right]\left\{d_{j}\right\} \cdot\left(J_{i} \omega\right)\right) \cos \left(J_{i} \omega t\right)\right. \\
& \left.+\left(\left[Y_{s j}^{i}\right]\left\{k_{j}\right\}-\left[Y_{c j}^{i}\right]\left\{d_{j}\right\} \cdot\left(J_{i} \omega\right)\right) \sin \left(J_{i} \omega t\right)\right],
\end{aligned}
$$

where

$$
\begin{aligned}
& {\left[Y_{c j}^{i}\right]=\left[\begin{array}{cccc}
x_{c j}^{i} & y_{c j}^{i} & 0 & 0 \\
0 & 0 & x_{c j}^{i} & y_{c j}^{i}
\end{array}\right] ;} \\
& {\left[Y_{s j}^{i}\right]=\left[\begin{array}{cccc}
x_{s j}^{i} & y_{s j}^{i} & 0 & 0 \\
0 & 0 & x_{s j}^{i} & y_{s j}^{i}
\end{array}\right] .}
\end{aligned}
$$

Then,

$$
\{F\}=\sum_{i=1}^{n}\left[\left\{F_{c}^{i}\right\} \cos \left(J_{i} \omega t\right)+\left\{F_{s}^{i}\right\} \sin \left(J_{i} \omega t\right)\right]
$$

where

$$
\begin{gathered}
\left\{F_{c}^{i}\right\}=\left[\begin{array}{llll}
Y_{c 1}^{i} & Y_{c 2}^{i} & \cdots & Y_{c l}^{i}
\end{array}\right]\left\{\begin{array}{c}
k_{1} \\
k_{2} \\
\vdots \\
k_{L}
\end{array}\right\} \\
+\left(J_{i} \omega\right)\left[\begin{array}{llll}
Y_{s 1}^{i} & Y_{s 2}^{i} & \cdots & Y_{s l}^{2}
\end{array}\right]\left\{\begin{array}{c}
d_{1} \\
d_{2} \\
\vdots \\
d_{L}
\end{array}\right\} \\
\left\{F_{s}^{i}\right\}=\left[\begin{array}{llll}
Y_{s 1}^{i} & Y_{s 2}^{i} & \cdots & Y_{s l}^{i}
\end{array}\right]\left\{\begin{array}{c}
k_{1} \\
k_{2} \\
\vdots \\
k_{L}
\end{array}\right\} \\
-\left(J_{i} \omega\right)\left[\begin{array}{lll}
Y_{c 1}^{i} & Y_{c 2}^{i} & \cdots \\
Y_{c l}^{i}
\end{array}\right]\left\{\begin{array}{c}
d_{1} \\
d_{2} \\
\vdots \\
d_{L}
\end{array}\right\} .
\end{gathered}
$$

Let

$$
\begin{aligned}
\{\widehat{k}\} & =\left\{\begin{array}{llll}
k_{1}^{T} & k_{2}^{T} & \cdots & k_{L}^{T}
\end{array}\right\} ; \\
\{\widehat{d}\} & =\left\{\begin{array}{llll}
d_{1}^{T} & d_{2}^{T} & \cdots & d_{L}^{T}
\end{array}\right\} ; \\
{\left[Y_{c}^{i}\right.} & =\left[\begin{array}{llll}
Y_{c 1}^{i} & Y_{c 2}^{i} & \cdots & Y_{c l}^{i}
\end{array}\right] ; \\
{\left[Y_{s}^{i}\right.} & =\left[\begin{array}{llll}
Y_{s 1}^{i} & Y_{s 2}^{i} & \cdots & Y_{s l}^{i}
\end{array}\right] .
\end{aligned}
$$

Now $\left\{F_{c}^{i}\right\}$ and $\left\{F_{s}^{i}\right\}$ could be simplified as

$$
\begin{aligned}
& \left\{F_{c}^{i}\right\}=\left[Y_{c}^{i}\right]\{\hat{k}\}+\left(J_{i} \omega\right)\left[Y_{s}^{i}\right]\{\widehat{d}\} \\
& \left\{F_{s}^{i}\right\}=\left[Y_{s}^{i}\right]\{\widehat{k}\}-\left(J_{i} \omega\right)\left[Y_{c}^{i}\right]\{\widehat{d}\} .
\end{aligned}
$$


Substituting (27) and (21) into (20) and decomposing $\cos \left(J_{i} \omega t\right)$ and $\sin \left(J_{i} \omega t\right)$ components,

$$
\begin{aligned}
{\left[\begin{array}{cc}
K-\omega^{2} M & \omega D \\
-\omega D & K-\omega^{2} M
\end{array}\right]\left\{\begin{array}{l}
U^{i} \\
V^{i}
\end{array}\right\}=\left\{\begin{array}{l}
h_{c}^{i}-F_{c}^{i} \\
h_{s}^{i}-F_{s}^{i}
\end{array}\right\} } \\
(i=1,2, \ldots, n) .
\end{aligned}
$$

Let

$$
A=\left[\begin{array}{cc}
K-\omega^{2} M & \omega D \\
-\omega D & K-\omega^{2} M
\end{array}\right] .
$$

Finally, the following equation could be obtained:

$$
\left\{\begin{array}{l}
U^{i} \\
V^{i}
\end{array}\right\}=A^{-1}\left\{\begin{array}{l}
h_{c}^{i}-F_{c}^{i} \\
h_{s}^{i}-F_{s}^{i}
\end{array}\right\} .
$$

The arbitrary node displacement of the shaft system could be expressed by the unbalanced force and oil-film force. The structural parameters of the shaft system are known; that is, matrix $A$ is known. The oil-film force is a function of journal bearing stiffness and damping coefficients. Hence, the genetic algorithm could be utilized to identify the oil-film dynamic characteristic parameters.

The genetic algorithm evaluates the chromosomes only according to the objective function. The objective of optimization is to find a group of parameters that make the square variances between the measured response and calculated response minimum:

$$
f=\sum_{i=1}^{n}\left[\left(U_{m}^{i}-U^{i}\right)^{2}+\left(V_{m}^{i}-V^{i}\right)^{2}\right]
$$

$$
(i=1,2, \ldots, n)
$$

where $n$ is the number of the harmonics.

There are 28 parameters that need to be identified, including the journal bearing stiffness and damping coefficients and residual unbalances. The individual chromosome string could be very long and the colony size very large because of too many parameters. Few methods are available to reduce the huge calculation costs. In one method, each parameter's search range could be set smaller according to experience and is so classified to ensure that the parameters obtained are available.

The main structural parameters for the practical water turbine generator set are given in Table 1. The identification results are given in Table 2 .

The journal guide bearing stiffness coefficients are identified and the supporting boundary condition of the generator set shaft system is found. Now, the modal characteristic of shaft system could be calculated by finite element modal analysis. The simulation results show that the first- and second-order natural frequencies are $4.23 \mathrm{~Hz}$ and $9.37 \mathrm{~Hz}$. The calculated first- and second-order natural frequencies matched quite well those obtained from modal parameter identification. This proves that the modal parameter and physical parameter identification methods utilized in this
TABle 1: Parameters of the generator set.

\begin{tabular}{lccc}
\hline \multicolumn{2}{c}{ Hydraulic turbine } & \multicolumn{2}{c}{ Electric generator } \\
\hline Outer diameter & $2.00 \mathrm{~m}$ & Upper shaft outer diameter & $1.39 \mathrm{~m}$ \\
Length & $4.98 \mathrm{~m}$ & Upper shaft length & $3.57 \mathrm{~m}$ \\
Turbine weight & $369 \mathrm{t}$ & Outer diameter & $2.20 \mathrm{~m}$ \\
Rated speed & $75 \mathrm{r} / \mathrm{min}$ & Length & $6.71 \mathrm{~m}$ \\
Runaway speed & $150 \mathrm{r} / \mathrm{min}$ & Rotor weight & $990 \mathrm{t}$ \\
\hline
\end{tabular}

paper are reasonable. Further, it can be confirmed that the unit would be steady because the first-order natural frequency is far from the rated frequency and runaway frequency.

\section{Conclusions}

(1) Modal parameter genetic time domain identification method proposed in this paper is suitable for larger structures or equipment that works only under environment excitation. The method has no influence on the normal operation of structures or equipment. The method makes the modal parameter identification of the running unit shaft system convenient. A new multifrequency signal decomposition technology to identify the main components of the measured signal and reject the image mode produced by noise has been used. This identification method offers a new study approach on the modal vibration characteristics of the rotating machine shaft system.

(2) The oil-film dynamic parameter genetic identification method proposed in this paper avoids the deficiency of traditional least square method, such as the need for many case responses, which is often unrealistic because oil-film dynamic parameters change greatly in different cases. Using the dynamic coefficients identified, the shaft system modal characteristics could then be studied by finite element method. The calculated first- and second-order natural frequencies matched quite well those obtained from modal parameter identification. It was proved that the modal parameter and physical parameter identification methods proposed in this paper are reasonable. The result of identification, that is, stiffness coefficients, could also provide a basis for the study of shaft system vibration response. Otherwise, because of the complex of the vibration sources loads, the work about the feed calculation of shaft system vibration response and contrast with the prototype test dates is being processed.

\section{Conflict of Interests}

The authors declare that there is no conflict of interests regarding the publication of this paper.

\section{Acknowledgments}

This research was supported by the Natural Science Foundation of China (51479165 and 51179154) and Program 2013KCT-15 for Shaanxi Provincial Key Innovative Research 
TABLE 2: Identification results of dynamic characteristic coefficients.

\begin{tabular}{lcccccc}
\hline & \multicolumn{2}{c}{ Stiffness coefficients $\left(10^{9} \mathrm{~N} / \mathrm{m}\right)$} & \multicolumn{4}{c}{ Damping coefficients $\left(10^{8} \mathrm{~N} \cdot \mathrm{s} / \mathrm{m}\right)$} \\
Guide bearing & Upper & Lower & Water & Guide bearing & Upper & Lower \\
\hline$K_{x x}$ & 0.95 & 7.85 & 1.26 & $C_{x x}$ & 2.82 & 10.03 \\
$K_{x y}$ & 0.46 & 5.43 & 0.89 & $C_{x y}$ & 0.78 & 3.51 \\
$K_{y x}$ & -0.53 & -5.51 & -0.94 & $C_{y x}$ & 0.86 & 3.74 \\
$K_{y y}$ & 1.25 & 8.19 & 1.52 & $C_{y y}$ & 5.94 & 1.04 \\
\hline
\end{tabular}

Team. Sincere gratitude is extended to the editor and anonymous reviewers for their professional comments and corrections, which greatly improved the presentation of the paper.

\section{References}

[1] Z. Wang, J. Yu, Y. Fang, X. Wen, J. Cao, and Q. Shi, "Characteristic analysis of rotor dynamics of large hydraulic generating unit," Journal of Hydroelectric Engineering, vol. 24, no. 4, pp. 6266, 2005.

[2] X. An, J. Zhou, L. Liu, and X. Xiang, "Lateral vibration characteristic analysis of the hydro generator set," Lubrication Engineering, vol. 33, no. 2, pp. 40-44, 2008.

[3] X. Xu, H. Hua, and Z. Chen, "Review of modal identification method based on ambient excitation," Journal of Vibration and Shock, vol. 21, no. 3, pp. 1-5, 2002.

[4] S. Li and J. Lian, "Genetic algorithm for hydraulic power house modal parameters identification," Journal of Tianjin University, vol. 42, no. 1, pp. 11-16, 2009.

[5] H.-D. Zhang, Y. Zhou, and J.-J. Lian, "Method based on ARMA model for modal parameter identification of a power house," Journal of Vibration and Shock, vol. 26, no. 5, pp. 115-118, 2007.

[6] P. Pennacchi, N. Bachschmid, A. Vania, G. A. Zanetta, and L. Gregori, "Use of modal representation for the supporting structure in model-based fault identification of large rotating machinery: part 1-theoretical remarks," Mechanical Systems and Signal Processing, vol. 20, no. 3, pp. 662-681, 2006.

[7] P. Mohanty and D. J. Rixen, "A modified Ibrahim time domain algorithm for operational modal analysis including harmonic excitation," Journal of Sound and Vibration, vol. 275, no. 1-2, pp. 375-390, 2004.

[8] Y. Guo, Y. Chen, J. Wang, and X. Yuan, "Principle and measure point research of bearing oil-film force identification," Lubrication Engineering, vol. 32, no. 8, pp. 39-41, 2007.

[9] D. Yao, W. Huang, and J. Zhou, "Identification of the journal bearing stiffness coefficients," Dynamic Engineering, vol. 25, no. 4, pp. 483-486, 2005.

[10] A. W. Lees and M. I. Friswell, "The evaluation of rotor imbalance in flexibly mounted machines," Journal of Sound and Vibration, vol. 208, no. 5, pp. 671-683, 1997.

[11] S. Edwards, A. W. Lees, and M. I. Friswell, "Experimental identification of excitation and support parameters of a flexible rotor-bearings-foundation system from a single run-down," Journal of Sound and Vibration, vol. 232, no. 5, pp. 963-992, 2000.

[12] R. Tiwari and V. Chakravarthy, "Simultaneous identification of residual unbalances and bearing dynamic parameters from impulse responses of rotor-bearing systems," Mechanical Systems and Signal Processing, vol. 20, no. 7, pp. 1590-1614, 2006.
[13] S. X. Zhao, H. Zhou, G. Meng, and J. Zhu, "Experimental identification of linear oil-film coefficients using least-meansquare method in time domain," Journal of Sound and Vibration, vol. 287, no. 4-5, pp. 809-825, 2005.

[14] V. Meruane and R. Pascual, "Identification of nonlinear dynamic coefficients in plain journal bearings," Tribology International, vol. 41, no. 8, pp. 743-754, 2008.

[15] X. Lou, Unbalance Identification Method Study for the Operating Rotor, Zhejiang University, Zhejiang, China, 1998.

[16] S. Bi, W. Jing, and N. Cui, "Recursive damping least square estimation of dynamic characteristic for journal bearings," Journal of Vibration Engineering, vol. 11, no. 4, pp. 410-415, 1998.

[17] R. Tiwari, A. W. Lees, and M. I. Friswell, "Identification of dynamic bearing parameters: a review," Shock \& Vibration Digest, vol. 36, no. 2, pp. 99-124, 2004.

[18] R. Tiwari, S. Manikandan, and S. K. Dwivedy, "A review of the experimental estimation of the rotor dynamic parameters of seals," Shock \& Vibration Digest, vol. 37, no. 4, pp. 261-284, 2005.

[19] X. Wang and L. Cao, Theory, Application and Software Implementation of Genetic algorithm, Xi'an Jiaotong University Press, Xi'an, China, 2002.

[20] G. Lu, X. Peng, and L. Zhang, Random Signal Process, Xi Dian University Press, Xi'an, China, 2002. 

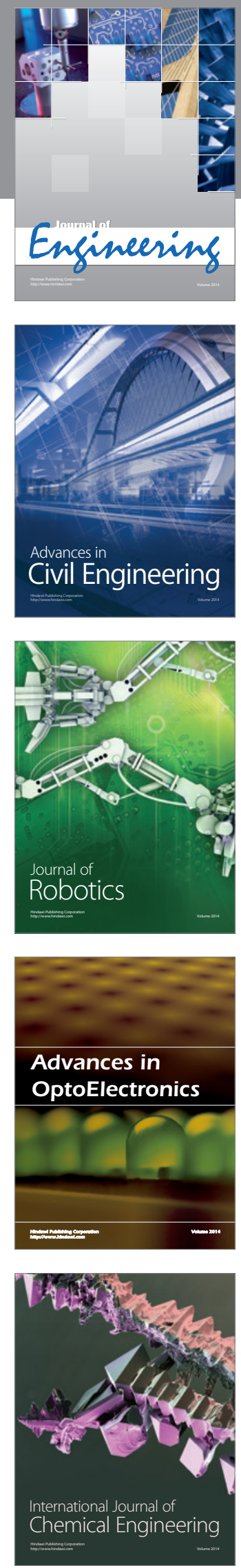

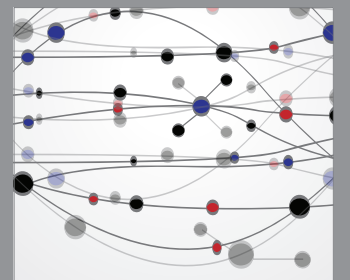

The Scientific World Journal
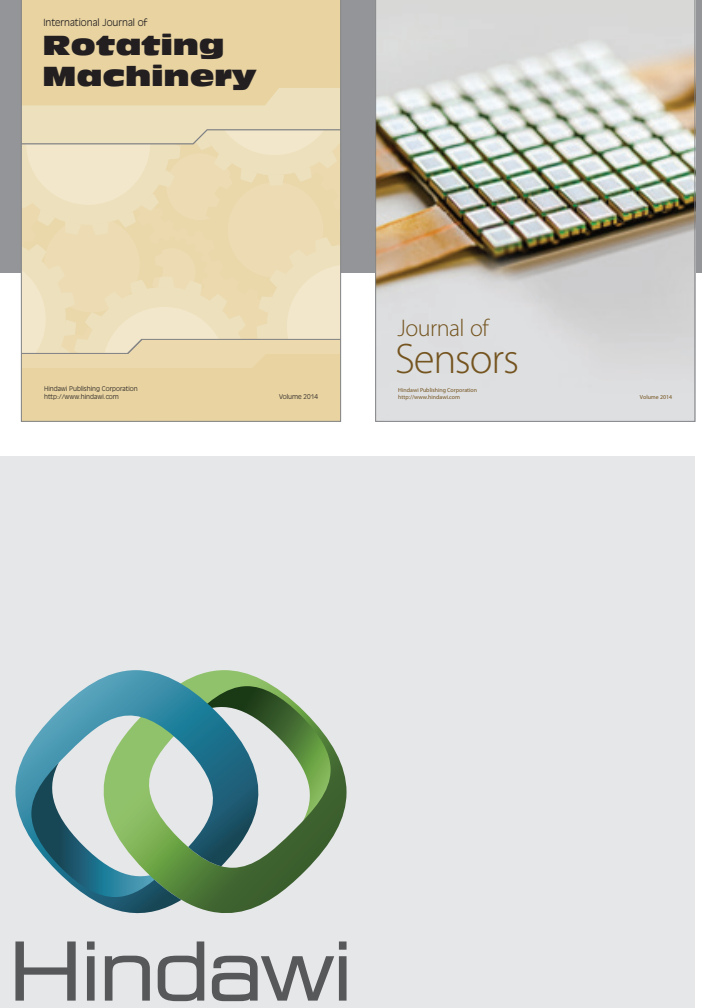

Submit your manuscripts at http://www.hindawi.com
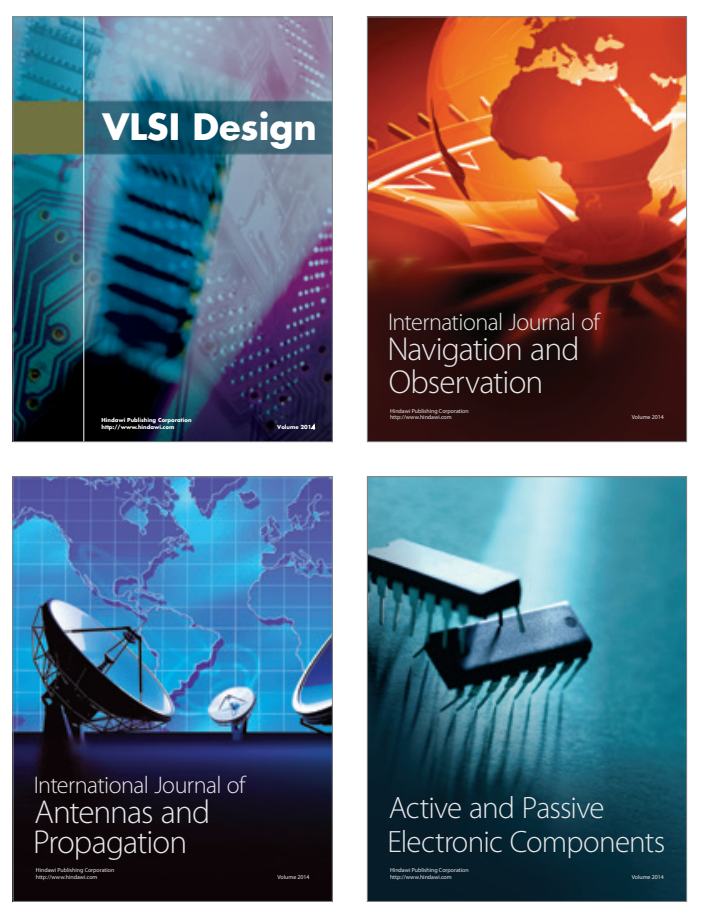
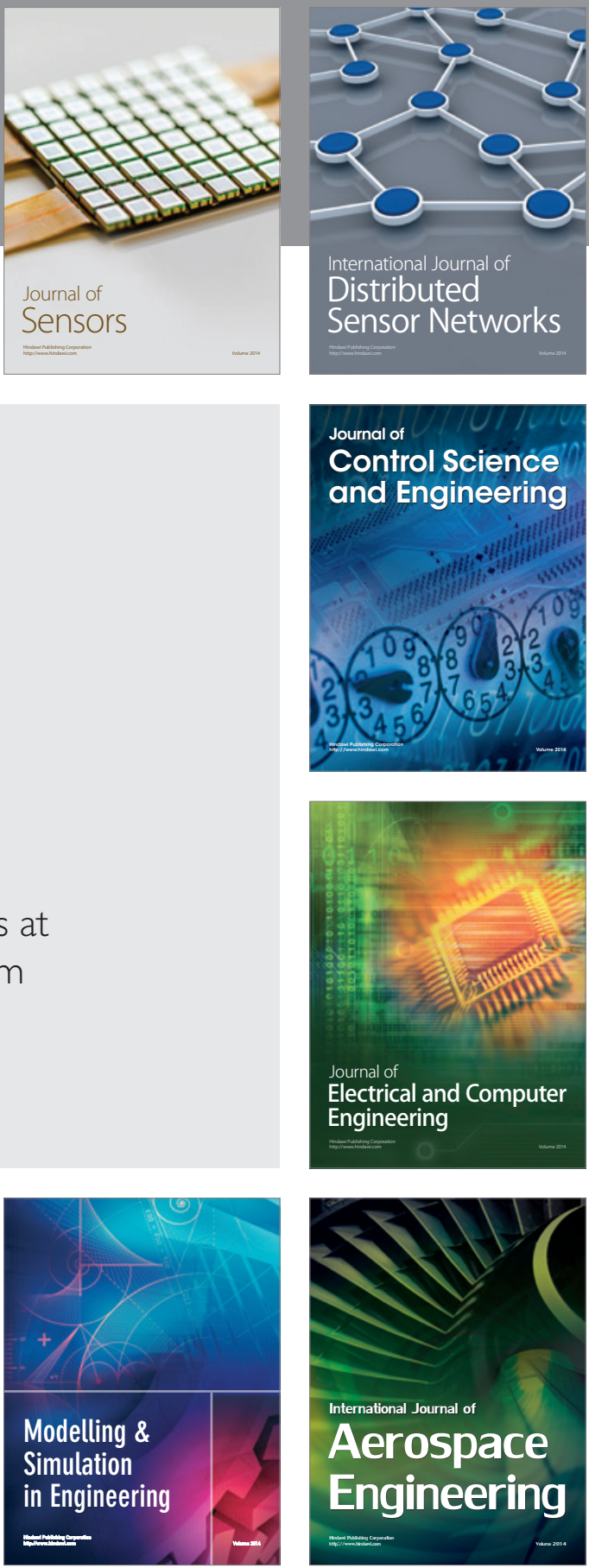

Journal of

Control Science

and Engineering
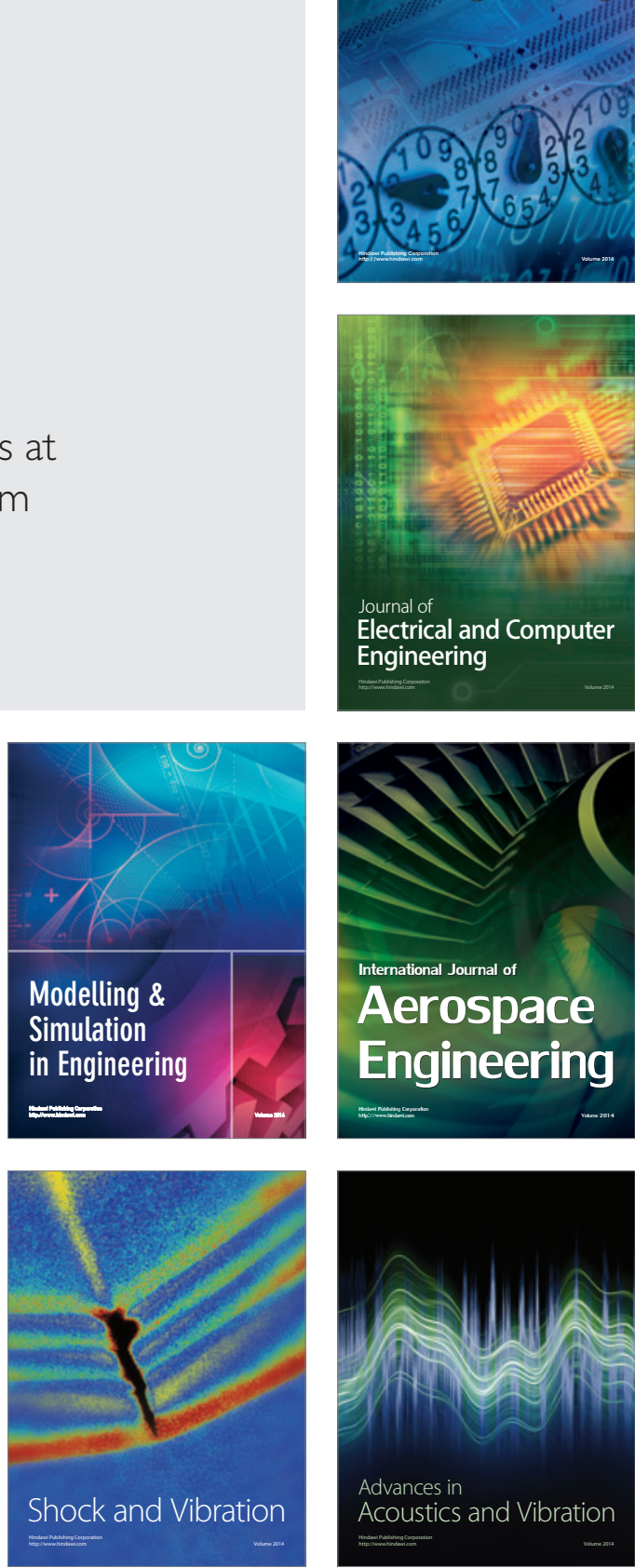